\title{
Bulk Acoustic Resonator Based on Piezoelectric ZnO Belts
}

\author{
Brent A. Buchine, ${ }^{\dagger}$ William L. Hughes ${ }^{\dagger}$ F. Levent Degertekin, ${ }^{\ddagger}$ and \\ Zhong L. Wang ${ }^{\star, \dagger}$
}

School of Materials Science and Engineering, Georgia Institute of Technology, Atlanta, Georgia 30332-0245, and George W. Woodruff School of Mechanical

Engineering, Georgia Institute of Technology, Atlanta, Georgia 30332-0405

Received February 15, 2006

\begin{abstract}
In this paper, a bulk acoustic resonator based on $\mathrm{ZnO}$ belts is demonstrated. This device shows a great deal of promise in applications as an electronic filter and as a mass sensor. The fabricated device was characterized using vector network analysis, and both the first and third harmonics of resonance were observed at approximately 247 and $754 \mathrm{MHz}$, respectively. A one-dimensional Krimholt-Leedom-Matthaei model was utilized to predict the resonant frequency of the device and confirm the observed behavior.
\end{abstract}

Bulk acoustic resonators (BAR) have shown a great deal of promise for applications as high-performance frequency control devices in wireless networks such as cell phones, navigation systems, satellite communication, and other forms of data communication. These devices consist of a thin piezoelectric film sandwiched between two electrodes. An RF signal applied through the thickness of the film produces mechanical motion, and fundamental resonance occurs when the film thickness equals $\lambda / 2$ the input signal. At the resonant frequency, the impedance of the device drops, whereas at anti-resonance, the impedance reaches a maximum. This behavior makes the resonator useful as an electronic filter. Subsystem miniaturization in filter applications is pushing toward on-chip components with low power consumption instead of the bulky off-chip quartz crystal and surface acoustic wave (SAW) devices currently being used. ${ }^{1,2}$ The most promising candidates for replacement are the solidly mounted resonator (SMR), ${ }^{3}$ thin-film bulk acoustic resonator (FBAR) ${ }^{4}$ and clamped-clamped beam resonator (beam resonator). ${ }^{5,6}$

In addition to applications in electronic circuits, BAR devices are immediately transferable as mass sensors. Saurbrey treated a change in mass applied to a resonator as a change in mass to the resonator itself. ${ }^{7}$ Since the resonant frequency of a BAR device is defined by its material properties and geometry, applying a voltage, mass, or temperature change to the resonator results in a shift of its characteristic frequency. The ultimate sensitivity of the sensor is determined by the device's quality factor $(Q)$ and modulus

\footnotetext{
* Corresponding author. E-mail: zhong.wang@mse.gatech.edu.

School of Materials Science and Engineering.

$\doteqdot$ George W. Woodruff School of Mechanical Engineering.
}

of elasticity. ${ }^{8,9}$ For the purpose of this paper, only resonator application will be discussed.

One challenge in manufacturing BAR devices is the achievement of a high-quality film. The piezoelectric materials currently being utilized are typically grown via sputter deposition. The results of this are low-quality films made up of oriented grains with a high concentration of defects. ${ }^{10}$ $\mathrm{ZnO}$ is particularly sensitive to temperature, acids, bases, and even water. As a result, other processes like cleaning, etching, metal layer patterning, passivation, reactive ion etching, and the stripping of photoresist all degrade the quality of the $\mathrm{ZnO} .{ }^{11}$ High-quality single crystals are desirable; however, microfabrication techniques result in poor material properties.

The bottom-up approach of nanotechnology has yielded many high-quality, single-crystal, and defect-free structures such as nanobelts. ${ }^{12}$ Piezoelectric nanobelts of $\mathrm{ZnO}$ become attractive in these applications because of their perfectly faceted, beam-like geometry, making them ideal candidates as SMR, FBAR, and beam resonators. ${ }^{13}$ However, handling belts can be cumbersome when attempting to manipulate these materials into useful devices. In addition, the current operational frequency range for devices that utilize electromechanical filters are between $200 \mathrm{MHz}$ and about $6 \mathrm{GHz}$. Films on the order of $500 \mathrm{~nm}$ to $15 \mu \mathrm{m}$ thick are desired in order to fabricate devices that operate within this range.

In this paper, we have utilized the process of nanotechnology to synthesize larger structures for the abovementioned radio frequency (RF) applications. Although the materials were synthesized using a bottom-up approach, traditional fabrication techniques were employed to manufacture other components of the resonator, such as the support 
substrate, ground pads, and contact electrodes. Vector network analysis was implemented to measure the scattering parameters $\left(S_{11}\right)$ and fully characterize the working device. ${ }^{14}$ A one-dimensional Krimholt-Leedom-Matthaei model (KLM) was also used to compare predicted resonant frequencies to those that were measured experimentally. ${ }^{15}$

The $\mathrm{ZnO}$ belts were grown using a single-zone tube furnace. The furnace was operated for a period of $12 \mathrm{~h}$ in order to increase the size of the belts per our application; the typical width dimensions of the belts varied between 5 and $30 \mu \mathrm{m}$, and the thickness ranged from a few hundred nanometers up to $5 \mu \mathrm{m}$ thick. In some cases, the belts were grown up to a centimeter long. As mentioned before, these large belts will resonate in a frequency range suitable for modern RF communication systems.

A (100) oriented silicon wafer was chosen as the support substrate for device fabrication. PECVD was used to deposit $400 \mathrm{~nm}$ of $\mathrm{Si}_{3} \mathrm{~N}_{4}$ onto the surface to provide an electrically insulating barrier between the device and the low-resistivity silicon substrate. Thermal evaporation was then used to deposit $200 \mathrm{~nm}$ of aluminum. An electron beam sensitive negative resist (ma-N2410) was spun onto the aluminum surface at $4000 \mathrm{rpm}$ to obtain a thickness of approximately $1 \mu \mathrm{m}$. The wafer was then cured at $90{ }^{\circ} \mathrm{C}$ on a hotplate for three minutes and subsequently diced into small chips approximately $1 \mathrm{~cm}^{2}$. A JEOL JSM-5910 SEM, modified with a J. C. Nabity Nanometer Pattern Generation System (NPGS), was used to pattern the contact electrodes for the device into the resist via e-beam lithography (patterns were designed using DesignCAD LT2000). The chip was subsequently developed with a $2.5 \%$ tetramethyl ammonium hydroxide (TMAH) solution for $90 \mathrm{~s}$ and rinsed with deionized water (DI). After the pattern was inspected with an optical microscope, a wet chemical aluminum etchant of acetic, phosphoric, and nitric acid was heated to $50{ }^{\circ} \mathrm{C}$ and used to remove the unwanted metal and define the large contact pads. The remainder of the resist was then dissolved in acetone and rinsed with 2-propanol (IPA) and DI. The electrodes were inspected again to ensure that the desired features were achieved and free from contamination (Figure 1a).

The next stage of processing involved the manipulation of the as-synthesized belts appropriately between the ground pads and the drive/sense electrode. First, $\mathrm{ZnO}$ belts were dispersed onto the surface of a clean silicon chip. A long thin glass capillary that had been pulled to a fine point was fixed to a set of Cascade Microtech MPH series micromanipulators. Under an optical microscope with a large working distance, the manipulators were operated carefully in order to identify a belt appropriate for testing. The capillary interaction forces between the tip of the glass rod and the belt resulted in a weak adhesion sufficient to lift the belt off of the surface of the silicon and redeposit it between the electrodes. Figure $1 \mathrm{~b}$ and $\mathrm{c}$ shows a belt after successful manipulation.

To deposit electrodes onto the side facets of the belt, an FEI NOVA dual-beam, focused ion beam microscope (FIB) was used. Typically, FIB is used for milling into a sample

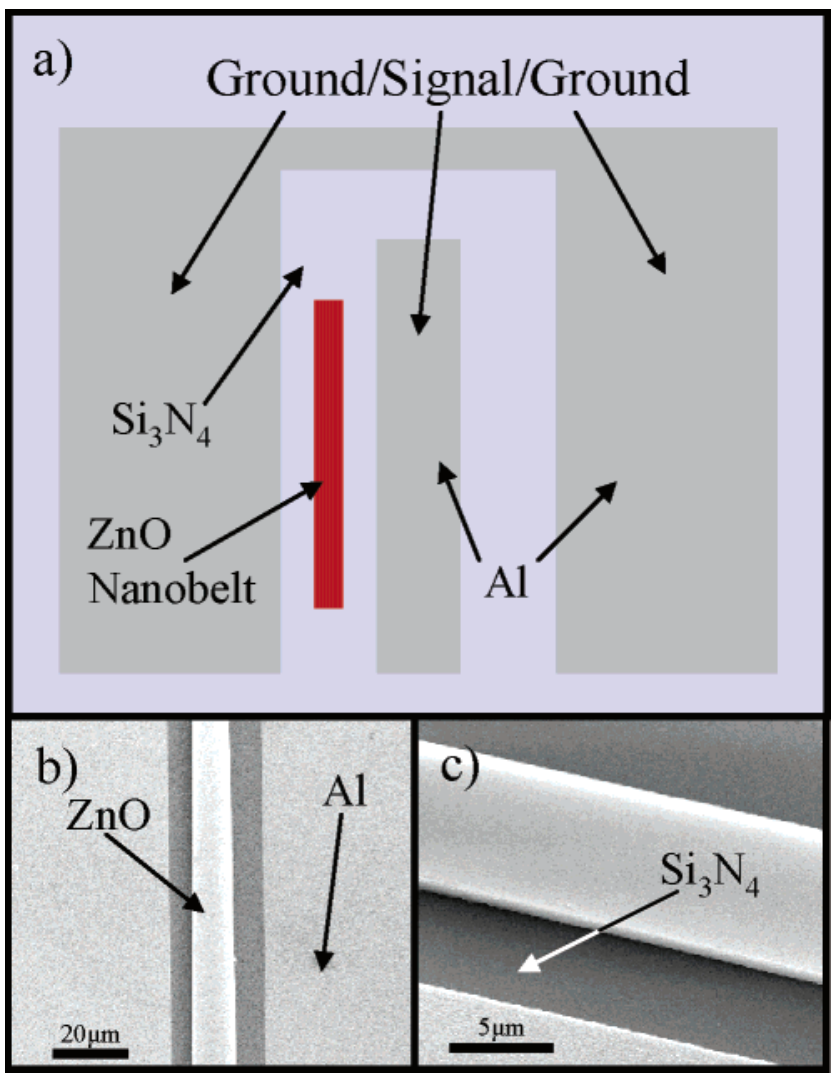

Figure 1. (a) Schematic of ground electrodes and position of belt prior to establishing contact. (b) SEM micrograph of belt between electrodes. (c) Close-up of side facets.

by accelerating gallium ions toward the sample's surface. Deposition of metal (instead of milling) can occur using an ion beam assisted CVD of an organometallic precursor. In our case, a methyl platinum gas was introduced into the chamber using a small capillary that suspends just above the sample. Gas molecules adsorb on the surface but only decompose into platinum metal $(\mathrm{Pt})$ where the ion beam interacts. A software package was used to control the position of the ion beam. ${ }^{16}$ The deposited Pt electrodes along with the lead wires that connect the belt to the contact pads can be seen in Figure 2.

For characterizing the device and measuring the resonant frequency of the $\mathrm{ZnO}$ belt-resonator, an Agilent ENA series 5071B network analyzer was used. Cascade Microtech Infinity ground-signal-ground (GSG) probes with a 150 $\mu \mathrm{m}$ pitch were utilized to make the on wafer measurements. Phase-matched K-Type $3.5 \mathrm{~mm}$ cables send the signal from the NA to the probes and back to the NA for analysis of the $\mathrm{S}_{11}$ parameters. To isolate the system and remove as many random errors as possible, the entire measurement was performed on a Herzan TS-140 active isolation table.

To ensure a high-quality signal, it is common to first calibrate the network analyzer using vector error correction. A calibration standard consisting of an open, short, and load (50 ohms) was used to quantify imperfections in the test equipment. The systematic errors were then stored in the network analyzer, and an error model was calculated to remove the effects from the subsequent measurement. This 


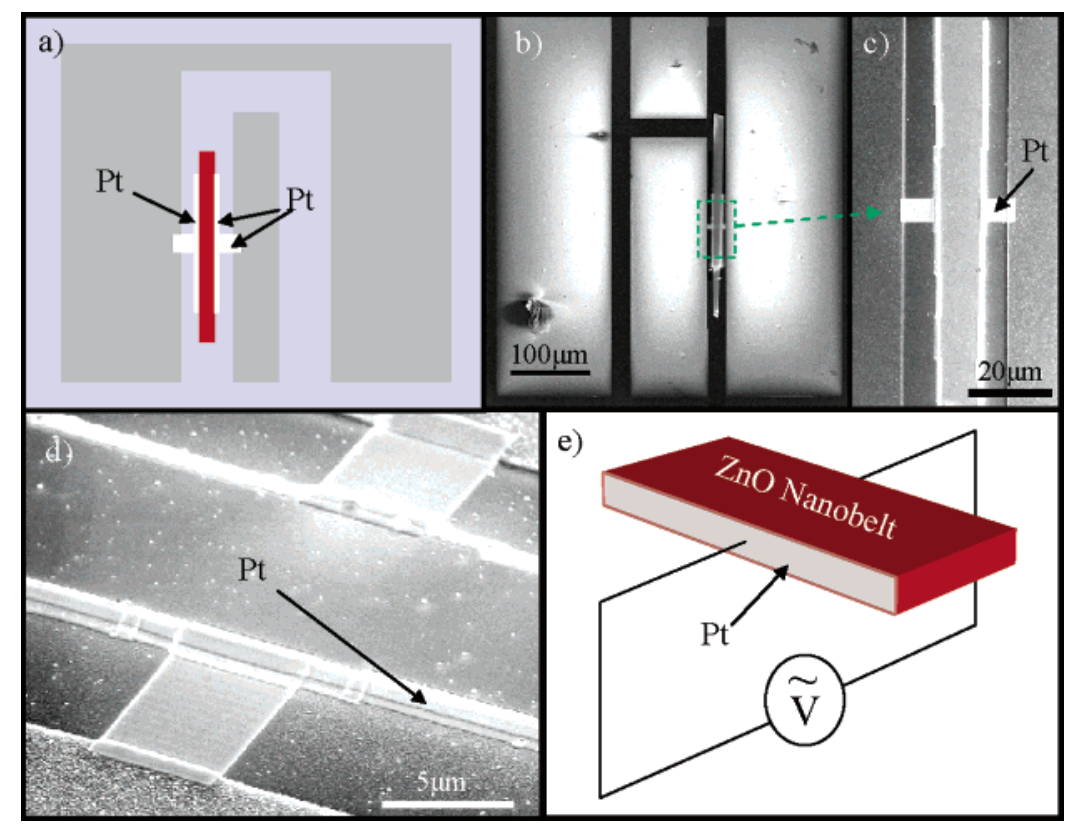

Figure 2. (a) Schematic of belt resonator with Pt electrodes. (b) Image of device prior to testing. (c) SEM micrograph of belt resonator. (d) Close-up of side facets and lead wires. (e) Depiction of stimulus applied through the thickness of the belt.

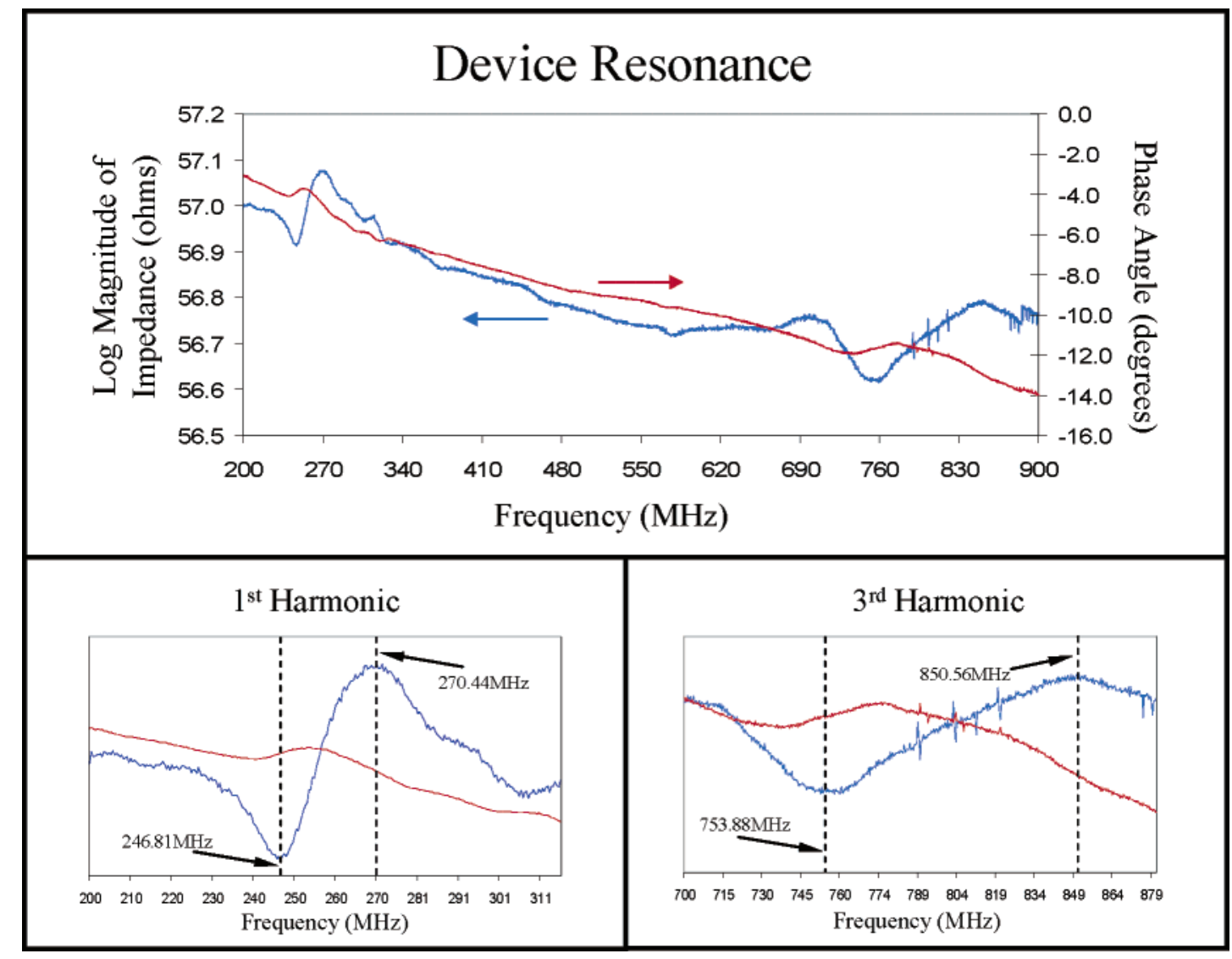

Figure 3. Plots of magnitude $(|Z|)$ and phase angle $(\theta)$ of impedance. The full frequency sweep from 200 to $900 \mathrm{MHz}$ is shown as well as the zoomed in portions of the 1 st and 3 rd harmonics.

enables a full calibration all the way to the probe tips. However, in addition to the testing equipment, additional systematic errors are introduced through contact resistance and pad parasitics of the fabricated device. An additional method, response calibration, was performed to remove any systematic errors created by these fixtures. To do this, a set of bare electrodes, absent a belt, was contacted and the data was stored in the network analyzer's memory. The trace was then displayed as data divided by memory to normalize the signal. The probes were then raised and positioned into 


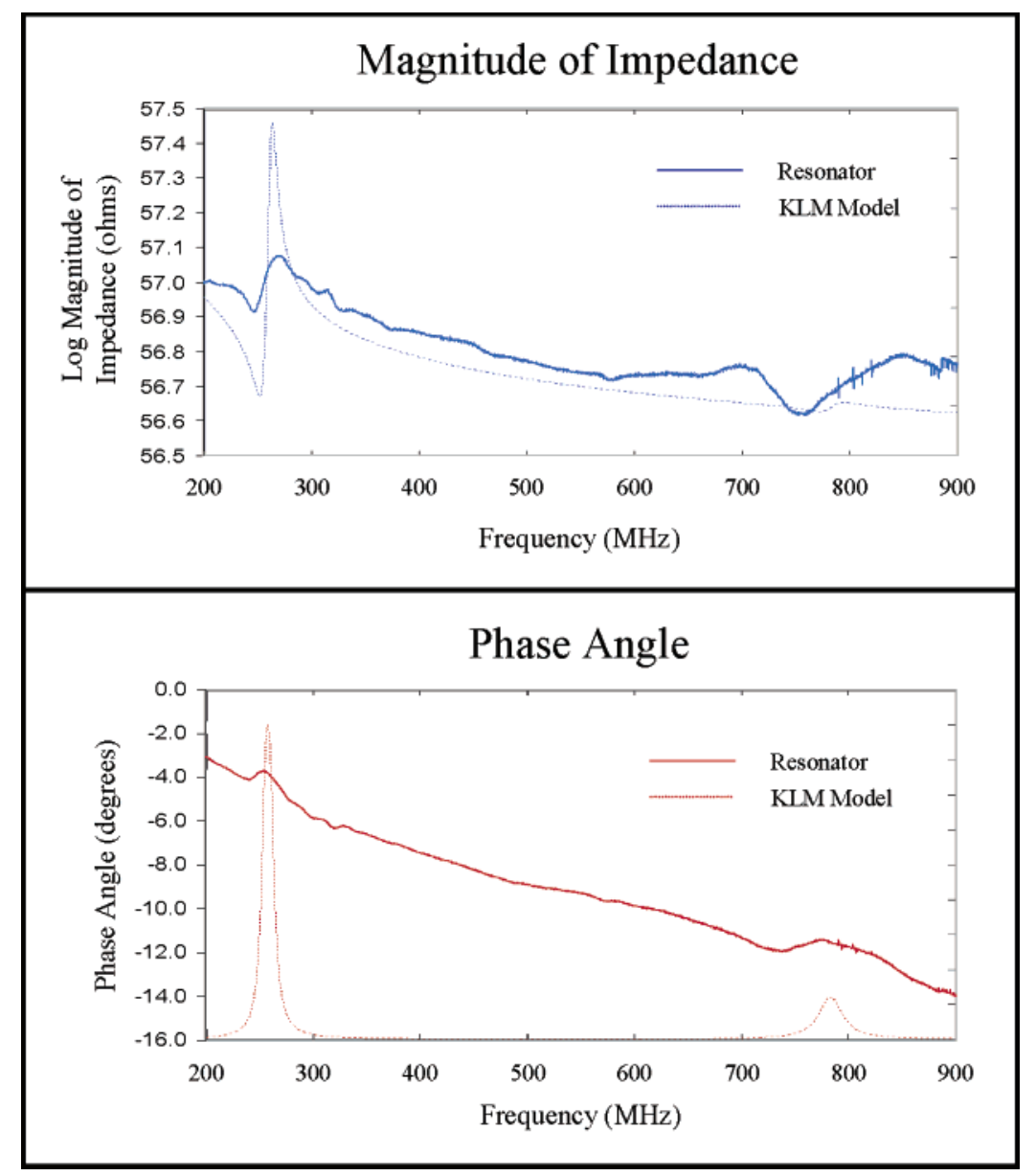

Figure 4. Predicted traces from the applied KLM model confirming the results from the working device.

\section{Resonant Frequency as a Function of Thickness}

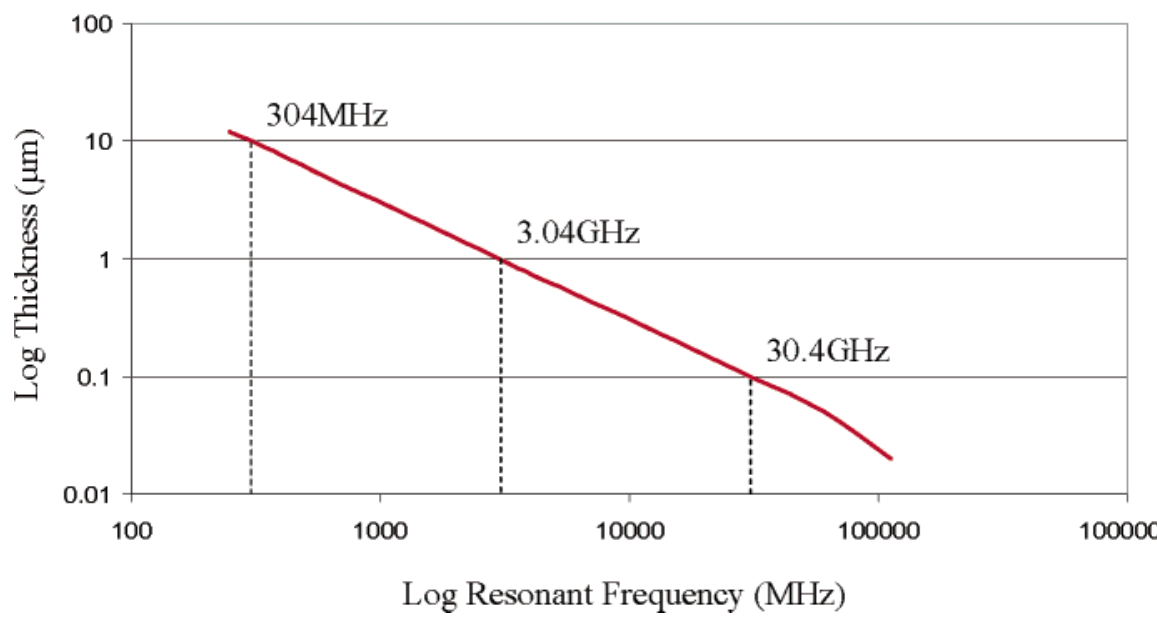

Figure 5. Calculated resonant frequency for belt resonators as a function of device thickness.

contact with the actual device. This additional process allowed calibration of the entire system all the way to the belt resonator. ${ }^{17}$ The resultant data is presented in Figure 3 .

The primary mode of resonance and anti-resonance is observed at 246.81 and $270.44 \mathrm{MHz}$, respectively. No quality factor $(Q)$ was calculated; however, it can be seen that the very large bandwidth at resonance and anti-resonance is the result of a lossy, low- $Q$ device. This results primarily from the interaction between the belt and the underlying substrate. Due to manipulation, the belt cannot be considered to be in intimate contact with the support substrate nor can it be considered to be free-standing. This quasi-substrate/belt interaction gives rise to an additional dampening mechanism other than the traditional dampening caused by ambient conditions in the environment. In addition, it can be seen that there is a taper in the belt. The width varies from 11.9 
$\mu \mathrm{m}$ at one end of the device to $12.3 \mu \mathrm{m}$ at the other. This asymmetry will broaden the resonant peak creating a resonance that is averaged across the thickness variation rather than defined at a specific dimension.

In addition to the first harmonic, we are also able to identify the third harmonic at a factor three times the primary peak $(753.88 \mathrm{MHz})$. It is common in lossy systems for subsequent harmonics to exhibit even lower $Q$ behavior, which is why the higher order mode appears even broader than the 1st; however, the existence of the third mode is a strong indication that the piezoelectric resonator is working properly. ${ }^{6}$

Devices fabricated via sputter deposition deal with loss in two ways. An SMR is typically fabricated on top of an acoustic reflector in order to prevent loss from being transmitted into the underlying silicon. In order for this to work, one must use three or four pairs of acoustically dissimilar materials. The FBAR deals with loss through the creation of an air/crystal interface. A free-standing device prevents interaction between the resonator and the underlying substrate. ${ }^{18}$ Fabrication of a completely free-standing belt device in order to reduce loss is currently in the works.

To ensure that the measured signal is coming from a working device, a one-dimensional KLM computer simulation was used to predict the resonant frequency based on a series of inputs. The parameters are thickness (chosen to be $12.1 \mu \mathrm{m})$, density, acoustic velocity, dielectric constant, coupling coefficient, quality factor, as well as source impedance of the test equipment (50 Ohms) and the impedance of the back layers, which were assumed to be air. All of the material properties were taken as bulk values for $\mathrm{ZnO}$. The results are displayed in Figure 4, and it can be seen that the theoretical results are in good agreement with the experimentally measured values.

Future work will involve the scaling down of the process to work with smaller nanobelts. To do this, it is important for one to first quantify how reducing the frequencydetermining dimension will shift the resonant frequency of the device. Where the resonant frequency is inversely proportional to thickness, ${ }^{19}$ it is possible to calculate the resonance of $\mathrm{ZnO}$ belts at a variety of dimensions. From a few select data points in Figure 5, it can be seen that ultrahigh resonance, in the $\mathrm{GHz}$ range, can be achieved as the device dimensions approach the nanoscale. In the future, expansion of the one-dimensional model in order to describe the resonant behavior of nanobelts in three dimensions will be pursued using finite element analysis. The other geometries described, like the beam resonator, the exploration of nanobelt SAW devices, and applications of piezoelectric cantilevers are included in future research. As these devices come to fruition, effects of voltage, mass, temperature, and pressure will be quantified and ultimately chemical sensing will be studied.

In conclusion, a new approach to resonator fabrication utilizing $\mathrm{ZnO}$ belts has been demonstrated. A union between the bottom-up synthesis process of nanotechnology and traditional fabrication techniques has resulted in the first ever belt resonator. A model was presented that explains the location of the resonant peaks as well as the breadth. Although the above design resulted in a low- $Q$ device, proof has been shown that the piezoelectric properties of $\mathrm{ZnO}$ belts can be exploited and future work will confirm the benefits of a high-quality single crystal.

Acknowledgment. Thanks to Prof. C. M. Lieber for stimulating discussions about this project. Thanks also to Reza Abdolvand for his comments on the usage of network analysis. This research is supported by the Defense Advanced Research Projects Agency (DARPA)-N66001-04-1-8903, the NASA Vehicle Systems Program, and Department of Defense Research and Engineering (DDR\&D).

\section{References}

(1) Lakin, K. M. IEEE Ultrason. Symp. 1999, 895-906.

(2) Nguyen, C. T. IEEE Int. Ultrason. Symp. 1995, 489-499.

(3) Dubois, M. A. MEMSWAVE 2003, July.

(4) Sue, Q. X.; Kirby, P.; Komuru, E.; Imura, M.; Zhang, Q.; Whatmore, R. IEEE Trans. Microwave Theory Tech. 2001 49, 769-778.

(5) DeVoe, D. L. Sens. Actuators, A 2001, 88, 263-272.

(6) Piazza, G.; Abdolvand, R.; Hoe, G. K.; Ayazi, F. Sens. Actuators, A 2004, 111, 71-78

(7) Suerbrey, G. Z. Phys. 1959, 155, 206-222.

(8) Yang, J.; Ono, T.; Esashi, M. Sens. Actuators, A 2000, 82, 102107.

(9) Funk, K.; Fabula, T.; Flik, G.; Larmer, F. J. Micromech. Microeng. 1995, 5, 143-146.

(10) Park, S. H.; Seo, B. C.; Yoon, G. J. Vac. Sci. Technol., A 2000, 18.

(11) Xu, T.; Wu, G.; Zhang, G.; Hao, Y. Sens. Actuators, A 2003, 104, $61-67$.

(12) Pan, Z. W.; Dai, Z. R.; Wang, Z. L. Science 2001, 291, 1947-1949.

(13) Hughes, W. L.; Wang, Z. L. Appl. Phys. Lett. 2003, 82, 2886-2888.

(14) Tilmans, H. A. C.; Untema, D. J.; Fluitman, J. H. J. Proc. Transducers '91, 1991, 533-537.

(15) Krimholt, R.; Leedom, D. A.; Matthaei, G. L. Electron. Lett. 1970, 6, 398-399.

(16) Giannuzzi, L. A.; Stevie, F. A. Introduction to Focused Ion Beams; Springer: New York, 2005.

(17) Agilent Application Note 1287-3, 2002.

(18) Ruby, R. C.; Bradley, P.; Oshmyansky, Y.; Chien, A. IEEE Ultrason. Symp. 2001, 813-821.

(19) Arnau, A. Piezoelectric Transducers and Applications; Springer: New York, 2004.

NL060351X 